\title{
Special issue on advances in image and video processing techniques
}

\author{
Mislav Grgic • Marta Mrak • Kresimir Delac
}

Published online: 29 January 2010

(C) Springer Science+Business Media, LLC 2010

\section{Background and motivation}

This special issue of the Multimedia Tools and Applications Journal contains extended papers selected from the image and video processing sessions and multimedia sessions of ELMAR symposia held in Zadar, Croatia, in 2008 and 2009 (more information about ELMAR symposia can be found here: www.elmar-zadar.org). Eight selected papers provide a coverage of recent advances in image and video processing. Papers were selected by the Guest Editors of this special issue in September 2009, and the respective authors were invited to submit the extended versions of their papers, along with further research they conducted on the subject.

Multimedia is all around us and we are often reminded that we live in the age of multimedia. It is in products and services like digital TV, IPTV, video and image databases, social networking etc. Time between research and final consumer product has never been shorter and this is one of the reasons why multimedia is still an attractive and widely researched area. Given the wide area of multimedia tools and applications usage in real life, it is necessary to research and develop algorithms and methods in various fields, and this special issue reflects this fact in its wide range of topics covered.

M. Grgic $\cdot$ K. Delac $(\bowtie)$

Faculty of Electrical Engineering and Computing, Department of Wireless Communications, University of Zagreb, Unska 3/XII, HR-10000 Zagreb, Croatia e-mail: kdelac@gmail.com

URL: http://www.vcl.fer.hr/kdelac/

M. Grgic

URL: http://www.vcl.fer.hr/mgrgic/

M. Mrak

Centre for Vision, Speech and Signal Processing, University of Surrey, GU2 7XH Guildford, Surrey, UK URL: http://personal.ee.surrey.ac.uk/Personal/M.Mrak/ 


\section{Scanning the special issue}

The two papers that open this special issue deal with an important subject of video quality assessment. The first paper is "Comparison of HDTV Formats using Objective Video Quality Measures" by Emil Dumic, Sonja Grgic and Mislav Grgic. By combining subjective and objective quality measures the authors show that progressive scanning should be preferred to interlaced for all future HDTV emissions. The second paper is "Foveated Mean Squared Error-A Novel Video Quality Metric" where Snjezana RimacDrlje, Mario Vranjes and Drago Zagar propose a new video quality measure called Foveated Mean Squared Error (FMSE) that takes into account the non-uniform sampling corresponding to the visual information acquisition process at the human retina. FMSE shows high correlation to the subjective quality assessment.

Segmentation is the central theme of the next three papers in this special issue. In "Segmentation of Optic Disc in Retinal Images using an Improved Gradient Vector Flow Algorithm" by Huiyu Zhou, Gerald Schaefer, Tangwei Liu and Faquan Lin, a novel medical image segmentation algorithm is presented. It is based on gradient vector flow and is used for segmenting the optic disc in retinal images to support automatic screening. Michal Kawulok addressed the problem of precise skin segmentation for sign language purposes in his article entitled "Energy-based Blob Analysis for Improving Precision of Skin Segmentation". The presented approach uses blob analysis and takes into account skin pixel topology which is usually disregarded by other color-based skin detectors. The paper "Automatic MPEG-4 Sprite Coding-Comparison of Integrated Object Segmentation Algorithms", by Alexander Glantz, Andreas Krutz, Thomas Sikora, Paulo Nunes and Fernando Pereira, proposes an automatic object segmentation algorithm for video sequences with a moving camera towards automatic object representation for sprite coding as standardized in MPEG-4 Visual. In their experiments, the authors have showed that their algorithm performs comparable to manually segmented groundtruth masks, implying almost perfect segmentation.

The last three papers cover areas of content retrieval, watermarking and face recognition. In the paper entitled: "Interactive Search and Browsing Interface for Large-scale Visual Repositories", Kan Ren, Risto Sarvas and Janko Calic present a way in which a user can intuitively navigate through a large image and video collections. In the paper "Two Dimensional Time-Frequency Analysis based Eigenvalue Decomposition Applied to Image Watermarking", Srdjan Stankovic, Irena Orovic, Nikola Zaric and Cornel Ioana propose applying spatial-frequency analysis and eigenvalue decomposition to provide an efficient image watermarking method. The efficiency of the proposed method and its robustness in the presence of various attacks is proven in extensive reported experiments. The last paper of this special issue is "Aligned Texture Map Creation for Pose Invariant Face Recognition" by Antonio Rama, Francesc Tarrés and Jürgen Rurainsky. The paper deals with a novel face recognition method called Partial Principal Component Analysis. The method has been proved to be efficient in pose-varying recognition scenarios. The paper also presents a novel approach for the automatic creation of 180 degrees aligned cylindrical projected face images using different views, which are then used for training purposes in the presented face recognition method.

Acknowledgements The Guest Editors would like to thank all those who have helped to make this special issue possible, especially to ELMAR symposium organizers and committee members, all authors and the reviewers. The Guest Editors would like to gratefully acknowledge Prof. Borko Furht, Editor-in-Chief of the Multimedia Tools and Applications Journal, for giving them the opportunity to publish this special issue. 


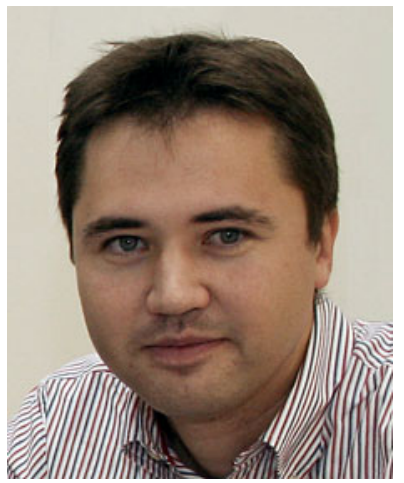

Mislav Grgic received B.Sc., M.Sc. and Ph.D. degrees in electrical engineering from the University of Zagreb, Faculty of Electrical Engineering and Computing, Zagreb, Croatia, in 1997, 1998 and 2000, respectively. He is currently an Associate Professor at the Department of Wireless Communications, Faculty of Electrical Engineering and Computing, University of Zagreb, Croatia. He participated in 5 scientific projects financed by the Ministry of Science, Education and Sports of the Republic of Croatia and 3 international COST projects of the European Commission. Currently, he is a project leader of the research project: "Intelligent Image Features Extraction in Knowledge Discovery Systems" financed by the Ministry of Science, Education and Sports of the Republic of Croatia and a member of the Management Committee of the European project COST Action IC0604. He published more than 110 papers in books, journals and conference proceedings in the area of image and video compression, content-based image retrieval, face recognition and digital mammography (computer-aided detection and diagnosis of breast cancer). Prof. Grgic is a senior member of IEEE.

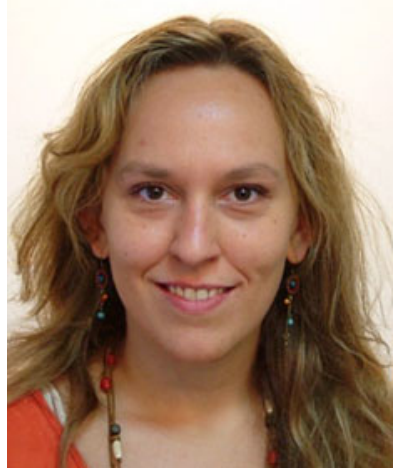

Marta Mrak received the Dipl. Ing. and MSc degrees in electronic engineering from the University of Zagreb, Croatia, in 2001 and 2003, respectively, and the PhD from Queen Mary University of London, UK, in 2006. Her main research interests are visual data processing, 2D/3D and high resolution video analysis and coding. She acts as a reviewer for several international conferences and journals, she contributed to EC FP6 projects aceMedia and VISNET II and has been acting as an expert reviewer for large research projects. She co-authored more than 60 publications including standardisation contributions and a patent. Dr Mrak is the recipient of the principal's award for best student work and has received two awards and the bronze medal from the University of Zagreb for the exemplary success during studies. She was awarded a German academic scholarship from DAAD in 2002. She has been a member of the organising/technical committees at several conferences, a co-chair of the IET VIE workshop on "Scalable coded media beyond compression" and she served as session chair and organiser of conference special sessions. She has contributed to MPEG and JPEG international standardisation activities. In 2009 she acted as guest co-editor for ELSEVIER journal of Signal Processing: Image Communication and in 2010 as co-editor of Springer book on High-Quality Visual Experience. 


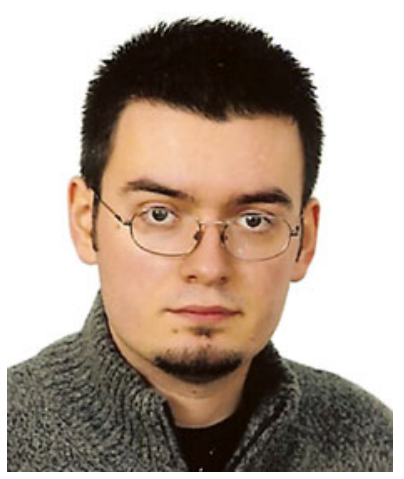

Kresimir Delac received his B.Sc.E.E degree in 2003, and Ph.D. degree in 2007 from University of Zagreb, Faculty of Electrical Engineering and Computing. He is a recipient of the silver medal "Josip Loncar" from the Faculty of Electrical Engineering and Computing in Zagreb for an outstanding Ph.D. thesis work. His research interests are image analysis and pattern recognition in biometric and medical applications. He is working as a consultant in a privately held company and is also affiliated to University of Zagreb, Faculty of Electrical Engineering and Computing, as a researcher on the project "Intelligent Image Features Extraction in Knowledge Discovery Systems" financed by the Ministry of Science, Education and Sports of the Republic of Croatia and as a guest lecturer. He is also a member of the Management Committee of the European project COST Action IC0604. 